\title{
Loss of Expression of the Tumor Suppressor CEACAM1 Links Different Hereditary Colorectal Carcinoma Sub- types to the Genesis of Sporadic Colorectal Carcinoma
}

\author{
Christian Neumaier $^{\mathrm{a}} \quad$ Stefanie Nittka $^{\mathrm{b}} \quad$ Michael Neumaier $^{\mathrm{b}}$ \\ ${ }^{a}$ Clinic of Radiation Oncology, ${ }^{b}$ Institute for Clinical Chemistry, Universitätsmedizin Mannheim, Medical Faculty Mannheim, University of \\ Heidelberg, Mannheim, Germany
}

\section{Keywords}

Colorectal cancer - HNPCC - CEACAM1 .

FCC-X $\cdot \beta$-Catenin - Microsatellite instability .

Microsatellite stability

\section{Summary}

According to their carcinogenesis, colorectal cancer (CRC) subtypes show distinct molecular parameters. Hereditary non-polypous colorectal cancer (HNPCC) is the most common inherited CRC characterized by clinical criteria and confirmed microsatellite instability (MSI). Interestingly, a recently identified subtype, familial colorectal cancer type $X$ (FCC-X), shows the same clinical criteria but microsatellite stability (MSS). CEACAM1 is a known tumor suppressor that regulates apoptosis in colon cells, and its loss is one of the most frequent events in early tumorigenesis of CRC. Therefore its loss may characterize precursor colon cells prior to neoplastic transformation. We analyzed tumor specimens of HNPCC and FCC-X patients in order to investigate whether there is a loss of CEACAM1 expression analogous to sporadic CRC and whether the expression of CEACAM1 would distinguish between these tumor entities. No differences in CEACAM1 expression were noted between HNPPC (n $=38)$ and FCC-X $(n=30)$ tumors. CEACAM1 was reduced in near-identical frequencies in 36/38 (95\%) HNPCC and 29/30 (97\%) FCC-X. This is the first report to demonstrate the loss of CEACAM1 expression in hereditary CRC. There was no difference between HNPCC and FCC-X. The frequency of expression loss was comparable to sporadic CRC, indicating that loss of CEACAM1 is an early event in colorectal tumorigenesis linking the genesis of sporadic and hereditary CRC.

\author{
Schlüsselwörter \\ Kolorektales Karzinom · HNPCC · CEACAM1 . \\ FCC-X · $\beta$-Catenin · Mikrosatelliteninstabilität · \\ Mikrosatellitenstabilität
}

\section{Zusammenfassung}

Bezüglich ihrer zugrunde liegenden Karzinogenese zeigen die Subtypen kolorektaler Karzinome (CRC) charakteristische molekulare Parameter. Das hereditäre nichtpolypöse CRC (HNPCC) stellt die größte Subgruppe der hereditären CRC dar, ist charakterisiert über klinische Kriterien und weist eine Mikrosatelliteninstabilität (MSI) auf. Interessanterweise zeigt ein nun identifizierter Subtyp, der familiäre CRC-Typ X (FCC-X), gleiche klinische Kriterien, aber eine Mikrosatellitenstabilität (MSS). CEACAM1 ist ein bekannter Tumorsuppressor, der Apoptose in Kolonzellen reguliert, und sein Expressionsverlust ist eine der am häufigsten vorkommenden Veränderungen in der frühen Genese von CRC. Daher könnte sein Expressionsverlust Kolonzellen vor deren neoplastischer Transformation charakterisieren. Wir analysierten Tumorgewebe von HNPCC- und FCC-X-Patienten, um herauszufinden, ob eine Analogie zum Expressionsverlust von CEACAM1 bei sporadischen CRC besteht und ob die Expression von CEACAM1 die untersuchten Entitäten unterscheiden kann. Es wurden keine Unterschiede bezüglich der Expression von CEACAM1 in Tumorgeweben von HNPCC $(n=38)$ und FCC-X $(n=30)$ festgestellt. CEACAM1 war in fast identischer Häufigkeit von 36/38 (95\%) in HNPCC und 29/30 (97\%) in FCC-X nachzuweisen. Dies ist der erste Bericht über den Verlust von CEACAM1 in hereditären CRC. Es konnte kein Unterschied zwischen HNPCC und FCC-X festgestellt werden. Die Häufigkeit des Verlustes ist vergleichbar mit der von CRC sporadischer Genese. Dies weist auf eine frühe Veränderung hin, die eine Verbindung zwischen hereditärer und sporadischer Genese herstellt.

\section{KARGER \\ Fax +497614520714 \\ Information@Karger.de}

www.karger.com (c) 2012 S. Karger GmbH, Freiburg

0378-584X/12/3510-0563\$38.00/0

Accessible online at:

www.karger.com/onk
Prof. Dr. med. Michael Neumaier

Institute for Clinical Chemistry, Universitätsmedizin Mannheim

Medical Faculty Mannheim, University of Heidelberg

Theodor-Kutzer Ufer 1-3, 68167 Mannheim, Germany

Tel. +49 621 383-2222, Fax -3819

christian.neumaier@umm.de 


\section{Introduction}

In Western civilizations, CRC represents one of the most prevalent cancers [1]. Over the last 20 years, genetic analyses of the hereditary CRC forms have generated important insights into the pathomechanisms of neoplastic transformation and progression that are also applicable to the sporadic CRC forms [2]. Firstly, defects of the Wnt/ $\beta$-catenin signaling pathway are responsible for the very rare familial adenomatous polyposis (FAP) and lead to tumors that show chromosomal instability but are stable in their microsatellite markers (microsatellite stability, MSS). Secondly, defects of the DNA mismatch repair (MMR) system are the molecular basis for the hereditary non-polypous colorectal cancers (HNPCCs) and will lead to microsatellite instability (MSI) and mutator phenotype, which is the hallmark of this entity. HNPCC is defined by the presence of germ-line mutations in any of the MMR genes (e.g. hMLH1, hMSH2 and, less frequently, MSH6 and PMS2). Traditionally, HNPCCs were identified by using clinical criteria (e.g. Amsterdam I, Amsterdam II, Bethesda) prior to immunohistochemical or/and genetic testing. Importantly, these defect patterns are closely linked to the pathobiochemistry of the highly prevalent sporadic CRC [3], implying that they are important for neoplastic growth and malignant progression. Indeed, analyses of chromosomal instability (CIN) and MSI tumors have shown remarkably consistent but largely different mutational spectra for the 2 tumor entities. For example, in CRC of the CIN type, neoplastic transformation most often starts with a loss of function of the $A P C$ gene, followed by mutations in other genes including kras, Smad4, DCC, and p53 [4]. In comparison, MMR defects will lead to subsequent instability within coding microsatellites that result in altered gene products of genes like TGFBR2, BRAF, IGF2R, PTEN, BAX, or Caspase-5 [5].

Recently, an additional entity of CRC termed familial colorectal cancer type X (FCC-X) has been identified among HNPCC patients as classified by the Amsterdam I and II or Bethesda criteria [6]. Up to $50 \%$ of the suspected patients classified by clinical criteria (especially Amsterdam criteria I) reveal an MSS without the presence of germ-line mutations, reflecting that the FCC-X type of CRC is related to the Lynch syndrome by clinical criteria, but is lacking the hallmarks of HNPCC regarding MSI and MMR proficiency [6]. Phenotypically, their histological features show resemblance to sporadic CRCs, i.e. later age of onset, preponderance of left-sided localization, lower incidence, and no mucin production [7-10]. Genes predisposing for FCC-X are currently unknown (reviewed in [11]).

CEACAMs are highly homologous adhesion molecules of the carcinoembryonic antigen (CEA) family. They are expressed in various human tissues, where they possess different biological functions [11]. In normal human colon mucosa, the expression of CEACAM1, CEACAM5 (formerly CEA) and CEACAM6 is limited to the differentiation zone of the crypts, where they represent a major constituent of the glycocalyx in the brush border. The transmembrane glycoprotein CEACAM1 has been shown to be a regulator of tissue homeostasis through differentiation-dependent apoptosis in the milk ducts of the human breast [12] and also in colon cells $[13,14]$. It has been known for years that CEACAM1 expression is lost in the great majority of all colorectal neoplastic lesions $[15,16]$. More recent studies have demonstrated that failure to express CEACAM1 during colonocyte differentiation occurs very early in colon tumorigenesis and, interestingly, equally frequent in neoplastic and hyperplastic tumor lesions $[12,13]$. Dysregulated expression in colon tumors has also been described for other CEACAMs [15-17], although their patterns and functions are less clear.

We investigated CEACAM1 expression in a group of HNPCC and FCC-X tumors from patients initially identified using the clinical Amsterdam I, II or Bethesda criteria by immunohistochemistry. The aim of our study was to evaluate whether expression of CEACAM1 can distinguish between HNPCC and FCC-X and also to compare the results from these hereditary tumors with respective data available from the highly prevalent sporadic CRC. A comparable rate of CEACAM1 loss would corroborate the notion that CRCs with different genetic defect cascades share a common ancestor and develop through genetic selection.

\section{Materials and Methods}

Paraffin-embedded tissues samples from patients classified according to clinical criteria were included in this study.

\section{Molecular MSI Analysis}

All samples were typed into HPCC and FCC-X using molecular and immunohistochemical methods. DNA from tumors and corresponding normal tissues was extracted and analyzed for MSI using a marker panel consisting of 7 different loci containing dinucleotide and 1 locus containing mononucleotide repeated sequences, as described by Chaves et al. [18]. MSI was graded as high (MSI-h) and stable (MSS) according to the numbers of positive markers (at least $30 \%$ and $0 \%$, respectively).

\section{Immunohistochemical Analyses}

Sections from paraffin-embedded tissue containing tumor and adjacent normal mucosa were examined. Antigen retrieval was done by standard microwave heating technique using either $10 \mathrm{mM}$ citric buffer $(\mathrm{pH} 6.0)$ for $10 \mathrm{~min}$ at $650 \mathrm{~W}$ or $1 \mathrm{mM}$ EDTA buffer ( $\mathrm{pH} \mathrm{8.0)}$ for $2 \mathrm{~min}$ at $650 \mathrm{~W}$, followed by $8 \mathrm{~min}$ at $170 \mathrm{~W}$. For the expression of MMR proteins, the following monoclonal antibodies (mab) were used in dilutions (v/v) as suggested by the manufacturers: anti-hMLH1 G168-728 (BD Pharmingen, Life Science Research, Heidelberg, Germany) diluted 1:240, antiPSM2 A16-4 (BD Pharmingen) diluted 1:300, anti-MSH2 FE 11 (Calbiochem, EMD, San Diego, CA, USA) diluted 1:80, and anti-MSH6 clone 44 (BD Biosciences, Life Science Research, Heidelberg, Germany) diluted 1:600. The accumulation of $\beta$-catenin was tested for using mab clone 14 (BD Bioscience, Heidelberg, Germany) diluted 1:2500. To assess apoptosis or proliferation in the tissue sections, mab M30 CytoDeath (Roche, Mannheim, Germany) diluted 1:50 or anti-Ki67 antibody MIB-1 (Dako Cytomation, Glostrup, Denmark) diluted 1:75 were used, respectively. For CEACAM1 and CEACAM5, mab 29H2 (Novocastra, Newcastle, 
UK) diluted 1:50 and mab T84.66 (kindly provided by Dr. J. Shively, Beckman Research Institute, City of Hope, Duarte, CA, USA) diluted to $1.25 \mu \mathrm{g} / \mathrm{ml}$ were used, respectively. For detection of primary antibody binding, we used the EliteABC kit (Vector Laboratories, Burlingame, CA, USA) according to the manufacturer's instructions. Immunohistochemical staining was evaluated semi-quantitatively by 2 independent investigators using conventional light microscopy. Expression of CEACAM1 or CEACAM6 was scored in comparison to adjacent mucosa as follows: no expression or strongly diminished '-', diminished '+', i.e. below $50 \%$ of normal staining intensity, normal ' ++ ', or ' +++ ' indicating overexpression. For CEACAM5, normal staining intensity was scored ' + '; increased or strongly increased staining was scored '++' or ' +++ ', respectively. Similarly, $\beta$-catenin staining was rated as absent '-', normal ' + ', elevated ' ++ ', and elevated with additional nuclear accumulation ' +++ ', For the evaluation of apoptosis, mab M30-stained mucosal cells from the upper half of longitudinal crypt sections were counted. The percentage of apoptotic cells was calculated separately for tumor lesions and corresponding normal tissue [13]. The proliferation labeling index was calculated as the percentage of Ki-67-positive cells in 1000 nuclei per tumor lesion in the lower crypt half, as described [13].
Statistical Analysis

For comparison of proliferation and apoptosis in FCC-X and HNPCC lesions, Welch's t-test was used.

\section{Results and Discussion}

HNPCC syndromes are identified by clinical criteria like Amsterdam 1 and 2 or Bethesda and represent the major group of inherited CRC predisposition with $4-5 \%$ of all CRCs [8]. HNPCCs are commonly characterized by MSI-h and MMR defects, but up to $40 \%$ [9] of these tumors do not display these hallmarks. These tumors termed FCC-X show differences in phenotypic appearance and are microsatellite stable due to their intact DNA mismatch repair capabilities [8-10]. Only in cases with questionable MMR status, screening for germ-line mutations of the MMR was done (data not

Table 1. Immunohistochemical evaluation of MMR protein expression (MLH1, MSH2 and MSH6) in HNPCC tumors ( $\mathrm{n}=38$ ) with MSI and FCC-X tumors $(\mathrm{n}=30)$ without underlying MSI (MSS)

\begin{tabular}{|c|c|c|c|c|c|c|c|c|c|c|c|c|c|}
\hline \multicolumn{7}{|l|}{ FCC-X } & \multicolumn{7}{|c|}{ HNPCC } \\
\hline Sample & MLH1 & MSH2 & MSH6 & MSI & $\begin{array}{l}\text { Age, } \\
\text { years }\end{array}$ & Sex & Sample & MLH1 & MSH2 & MSH6 & MSI & $\begin{array}{l}\text { Age, } \\
\text { years }\end{array}$ & Sex \\
\hline S-01 & + & + & + & stable & 46 & $\mathrm{M}$ & H-01 & - & + & + & high & n.s. & n.s. \\
\hline S-02 & + & + & + & stable & 52 & $\mathrm{~F}$ & $\mathrm{H}-02$ & + & - & - & high & 49 & M \\
\hline S-03 & + & + & + & stable & 34 & $\mathrm{~F}$ & $\mathrm{H}-03$ & - & + & + & high & 39 & M \\
\hline S-04 & + & + & + & stable & 64 & M & $\mathrm{H}-04$ & - & + & + & high & 43 & M \\
\hline S-05 & + & + & - & stable & 41 & $\mathrm{~F}$ & $\mathrm{H}-05$ & + & - & - & high & 42 & M \\
\hline S-07 & + & + & + & stable & 48 & M & $\mathrm{H}-07$ & - & + & + & high & 24 & $\mathrm{~F}$ \\
\hline S-08 & + & + & + & stable & 54 & M & $\mathrm{H}-08$ & - & + & - & high & 47 & M \\
\hline S-09 & + & + & + & stable & n.s. & n.s. & H-09 & + & - & - & high & 58 & $\mathrm{~F}$ \\
\hline S-10 & + & + & + & stable & n.s. & n.s. & H-10 & + & - & - & high & 58 & $\mathrm{~F}$ \\
\hline S-11 & + & + & + & stable & n.s. & n.s. & H-11 & + & + & + & high & 46 & M \\
\hline S-12 & + & + & + & stable & 29 & $\mathrm{~F}$ & H-12 & - & + & + & high & n.s. & $\mathrm{F}$ \\
\hline S-13 & + & + & + & stable & 44 & M & H-13 & + & - & - & high & 40 & $\mathrm{~F}$ \\
\hline S-14 & + & + & + & stable & 40 & $\mathrm{~F}$ & H-14 & - & + & + & high & 32 & M \\
\hline S-17 & + & + & + & stable & 70 & $\mathrm{M}$ & H-17 & - & + & + & high & 42 & M \\
\hline S-18 & + & + & - & stable & 29 & M & H-18 & - & + & + & high & 32 & M \\
\hline S-19 & + & + & + & stable & 56 & $\mathrm{~F}$ & H-19 & - & + & + & high & 32 & $\mathrm{~F}$ \\
\hline S-20 & + & + & + & stable & 36 & $\mathrm{~F}$ & H-20 & - & + & + & high & n.s. & n.s. \\
\hline S-21 & + & + & + & stable & 48 & M & H-21 & + & + & - & high & 56 & $\mathrm{~F}$ \\
\hline $\mathrm{S}-22$ & + & + & + & stable & 38 & M & H-22 & + & + & - & high & 41 & M \\
\hline S-23 & + & + & + & stable & 46 & M & H-23 & + & - & + & high & n.s. & n.s. \\
\hline S-24 & + & + & + & stable & 26 & $\mathrm{~F}$ & H-24 & - & + & + & high & n.s. & n.s. \\
\hline S-25 & + & + & + & stable & 46 & $\mathrm{~F}$ & H-25 & - & + & + & high & 39 & $\mathrm{M}$ \\
\hline S-26 & + & + & + & stable & 70 & M & H-26 & - & + & + & high & 44 & $\mathrm{~F}$ \\
\hline S-27 & + & + & + & stable & 39 & $\mathrm{~F}$ & H-27 & + & - & + & high & 40 & $\mathrm{M}$ \\
\hline S-28 & + & + & + & stable & 31 & M & H-28 & - & + & + & high & 42 & M \\
\hline S-29 & + & + & + & stable & 61 & M & H-29 & - & + & + & high & 39 & M \\
\hline S-30 & + & + & + & stable & 40 & M & H-30 & - & + & + & high & 43 & $\mathrm{~F}$ \\
\hline & & & & & & & H-31 & + & - & + & high & 46 & $\mathrm{~F}$ \\
\hline & & & & & & & H-32 & - & + & + & high & 48 & M \\
\hline & & & & & & & H-33 & + & - & + & high & 45 & $\mathrm{~F}$ \\
\hline & & & & & & & H-36 & - & + & + & high & 39 & M \\
\hline & & & & & & & H-37 & + & - & - & high & 47 & M \\
\hline & & & & & & & H-38 & + & + & + & high & 46 & M \\
\hline
\end{tabular}

Overall, 35 of the 68 patients are male (M), 25 female (F), and 8 are not specified (n.s.). The median age is 42.5 years for HNPCC and 42.0 years for FCC-X. 

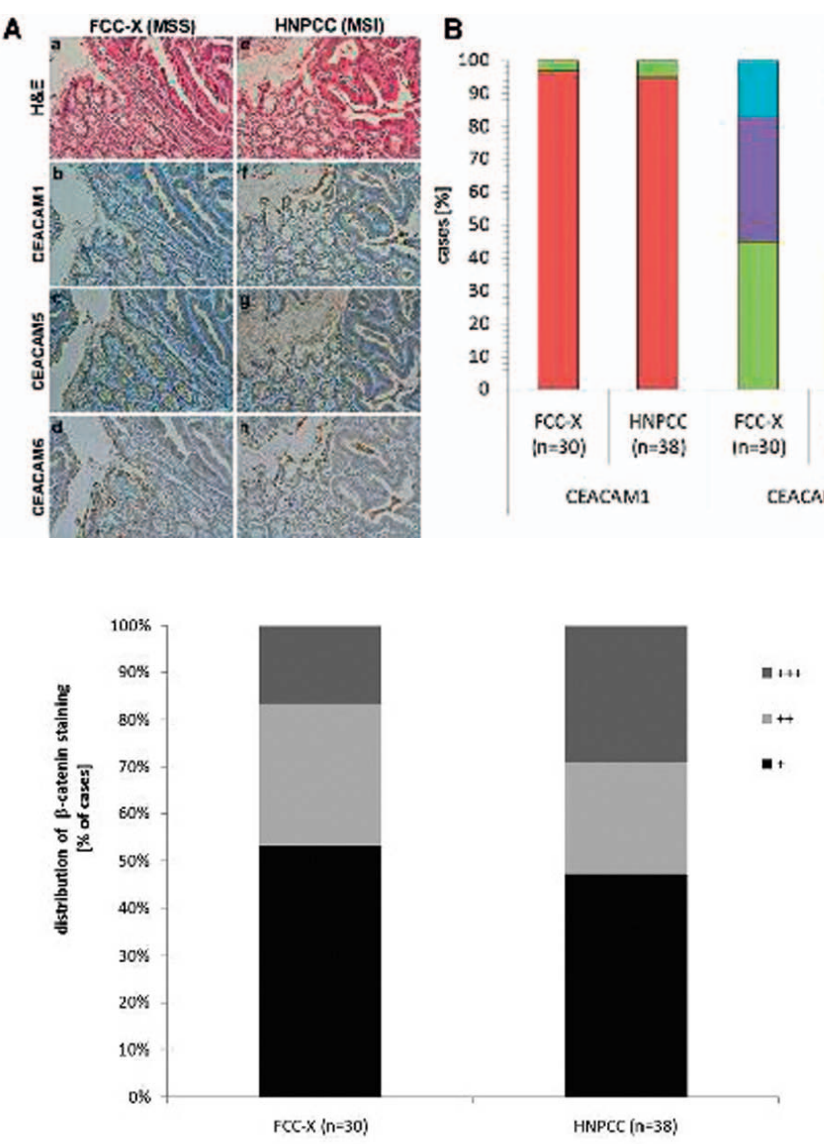

Fig. 2. Comparison of $\beta$-catenin staining intensities in tissue sections of FCC-X and HNPCC tumors. Regular staining was scored as ' + ', increased staining as ' ++ ', and increased staining with additional nuclear accumulation was scored as ' +++ '. For 1 HNPCC tumor, $\beta$-catenin staining could not be performed.

shown). Final classification of these tumors was done regarding their germ-line status. Tumors displaying no alterations were classified into the FCC-X group and tumors harboring alterations were assigned to the HNPCC group. In our study of 68 clinically verified HNPCC cases, we found $44 \%$ to resemble FCC-X-type CRC $(\mathrm{n}=30)$ whereas 38 cases were HNPCCs classified MSI-h on the basis of either microsatellite status or expression of hMLH1, hMSH2 and hMSH6 (table 1).

All FCC-X tumors were microsatellite stable, but we found loss of hMSH6 expression in 2 cases. These 2 FCC-X cases with no proven germ-line mutation in MMR are thought to accumulate hMSH6 deficiency by accident, not as a 'driver mutation'. In contrast, the lesions of the HNPCC group with MSI-h showed MMR deficiencies in a heterogeneous way, with either loss of hMLH1 or the combined loss of hMSH2 and hMSH6 expression. In 1 tumor (2.6\%), expression of hMLH1, hMSH2 and hMSH6 could be demonstrated. We did not exclude rarely occurring mutations in PSM2 or other MMR-related genes. Alternatively, the classification of this tumor as HNPCC may have been unjustified and emphasizes the importance to evaluate other markers in order to classify the nature of hereditary tumors in these cases [7].

The dysregulation of the CEACAM family members during colon tumorigenesis is very common, affecting all members of the gene family expressed in colon mucosa. Higher expression of CEACAM6 is observed in hyperplastic polyps and adenomas [17] and has indeed been reported in the earliest report on CEACAM dysregulation in the majority of CRCs [15]. In our study, CEACAM6 expression is increased in $34.2 \%$ and $33.3 \%$ of HNPCC and FCC-X tumors, respectively (fig. 1). CEACAM5 expression is unchanged in $37.9 \%$ of the FCC-X lesions and in $47.3 \%$ of the HNPCCs, while it is overexpressed in $17.2 \%$ and $13.2 \%$, respectively. We noted a tendency to decreased CEACAM5 expression in $44.9 \%$ compared to $39.5 \%$ of the cases in FCC-X tumors or HNPCCs, respectively. The variant expression levels of CEACAM5 confirmed earlier reports in sporadic CRC [15]. These data concur with comparisons between HNPCC tumors and the highly prevalent sporadic MSS CRC, revealing no impact of MMR defects on CEACAM5 expression [19]. Finally, the loss of CEACAM1 is the most pronounced observation in HNPCC and FCC-X. In comparison to other colon tumors of both hyperplastic and neoplastic origin so far investigated [13, 15-17], we now demonstrate that the expression of CEACAM1 is affected in these hereditary tumors at the same frequencies (fig. 1). Indeed, 96.7\% (29/30) of the FCC-X and $94.7 \%(36 / 38)$ of the HNPCC cases showed a severe decrease or complete loss of CEACAM1.

These results demonstrate for the first time that the alterations in the expression of CEACAMs are basically indistinguishable between hereditary CRC tumors that have developed from different genetic defects. We also conclude that no differences exist between the sporadic CRCs and the hereditary forms investigated with respect to both frequencies and grades of CEACAM1 loss. Furthermore, no differences exist between the CRCs classified as HNPCC, whether of MSI-h character or FCC-X. It is reasonable to assume that mucosal CEACAM1 expression would also be missing in the hereditary FAP syndrome, rare tumors sharing with the sporadic CRC the defects of the Wnt signaling pathway as their under- 
Fig. 3. (A) Detection of apoptotic cells based on M30 immunohistochemistry staining. The number of positive cells for both normal mucosa (NM) and tumors (T) was counted separately. (B) Proliferation in FCC-X and HNPCC tumors was detected by staining for Ki-67 antigen. Due to insufficient tissue, Ki-67 staining could not be performed for all HNPCC tumors. Differences in proliferation did not reach statistical significance based on Welch's t-test $(\mathrm{p}=0.1452)$.
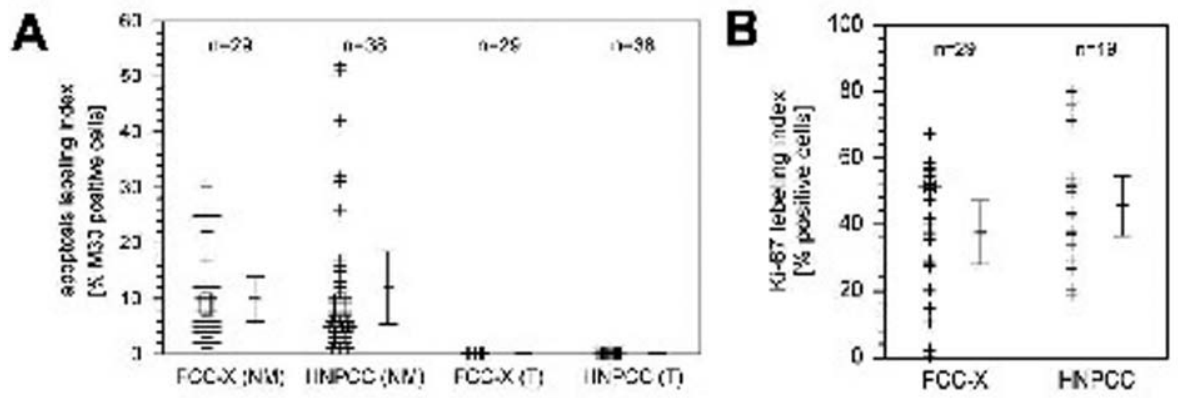

lying pathomechanism of the CIN type. Specifically, CEACAM1 expression is not different between Wnt-/APCdefective sporadic CRCs and tumors expressing APC on the basis of $\beta$-catenin overexpression [13]. Our findings of alterations in CEACAM expression support the idea of molecular similarities between sporadic MSS, CRC and FCC-X, as shown for the RAS/RAF and Wnt pathway by Sanchez-deAbajo et al. [20]. Additionally, our results confirm that hereditary-type FCC-X and HNPCC CRCs show low levels of $\beta$-catenin accumulation [20] (fig. 2). Due to the distribution pattern of the $\beta$-catenin expression, we can conclude that the role of $\beta$-catenin within the underlying pathway is less important than the CEACAM dysregulation for the carcinogenesis of HNPCC and FCC-X tumors. In any case, the $\beta$-catenin immunohistochemical analysis confirms that deregulation of the Wnt pathway is not a common carcinogenic mechanism in these subgroups of tumors. Taken together, we have shown that the alterations in the expression of CEACAMs, most notably of CEACAM1, occur in different types of hereditary CRC with the clinical phenotype of HNPCCs. Together with the data in the literature, this confirms and also extends the possible role of CEACAM1 in tumor generation as presented in recent reports about CEACAM1 as important regulator of, presumably differentiation-dependent, apoptosis [12-14]. For proliferation and apoptosis, we could not detect significant differences between the tumor entities analyzed in our study (fig. 3). The $\mathrm{p}$ values of the 2-sided Welch t-test for proliferation and apoptosis were $\mathrm{p}=0.15$ and $\mathrm{p}=0.63$, respectively.

The failure to express the cell membrane adhesion molecule CEACAM1 already in the great majority of earliest, microscopic hyperplastic colon crypt lesions has led to the proposal of a modified genetic multi-step (Vogelstein) model of colorectal carcinogenesis [13]. In this recent model, it has been proposed that failure to express CEACAM1 is an important contributor to hyperplastic tumor development. These tumor forms almost invariably regress due to non-permissive, i.e. lethal, molecular defects. However, neoplastic transformation can be selected for in very rare cases through the contraction of critical, i.e. permissive, genetic or epigenetic defects in the Wnt signaling pathway or the MMR system. The data presented in this study support this hypothesis. They show that CEACAM1 loss is the common denominator linking CRCs of very distinct genetic make-up. The causes for the dysregulation of CEACAM expression and loss of differentiation-dependent apoptosis are not known. Further investigations are imperative to answer this question and to clarify the concept of permissive mutations under the condition of CEACAM1 down-regulation as a pacemaker of colorectal tumor development.

\section{Acknowledgements}

We thank Prof. Magnus von Knebel-Doeberitz, Institute of Pathology, University of Heidelberg, and Prof. Christopher Poremba, Institute of Pathology, Heinrich-Heine Universität Düsseldorf, for kindly providing tissue samples of HNPCC and FCC-X tumors from their pathology archive.

\section{Disclosure Statement}

All authors state that there are no conflicting interests. There is no external funding.

\section{References}

$>_{1}$ Becker N, Altenburg HP, Stegmaier C, Ziegler H: Report on trends of incidence (1970-2002) of and mortality (1952-2002) from cancer in Germany. J Cancer Res Clin Oncol 2007;133:23-35.

$\checkmark 2$ Fearon ER, Vogelstein B: A genetic model for colorectal tumorigenesis. Cell 1990;61:159-167.
Kinzler KW, Vogelstein B: Lessons from hereditary colorectal cancer. Cell 1996;87:159-170.

4 Fearnhead NS, Wilding JL, Bodmer WF: Genetics of colorectal cancer. Br Med Bull 2002;64:27-43.

$\checkmark 5$ Chao EC, Lipkin SM: Molecular models for the tissue specificity of DNA mismatch repair-deficient carcinogenesis. Nucleic Acids Res 2006;34:840-852.
6 Lindor NM, Rabe K, Petersen GM, et al.: Lower cancer incidence in Amsterdam-I-criteria families without mismatch repair deficiency: familial colorectal cancer type X. JAMA 2005;293:1979-1985.

7 Abdel-Rahman WM, Peltomäki P: Lynch syndrome and related familial colorectal cancer. Crit Rev Oncog 2008;14:1-22; discussion 23-31. 
8 Jass JR: Hereditary non-polyposis colorectal cancer: the rise and fall of a confusing term. World J Gastroenterol 2006;12:4943-4950.

9 Valle L, Perea J, Carbonell P, et al.: Clinicopathologic and pedigree differences in Amsterdam Ipositive hereditary nonpolyposis colorectal cancer families according to tumor microsatellite instability status. J Clin Oncol 2007;25:781-786.

10 Llor X, Pons E, Xicola RM, et al.: Differential features of colorectal cancer fulfilling Amsterdam criteria without involvement of the mutator pathway. Clin Cancer Res 2005;11:7304-7310.

11 Hammarström S: The carcinoembryonic antigen (CEA) family: structures, suggested functions and expression in normal and malignant tissues. Semin Cancer Biol 1999:9:67-81.

12 Kirshner J, Chen CJ, Liu P, Huang, Shively JE: CEACAM1-4S, a cell-cell adhesion molecule, mediates apoptosis and reverts mammary carcinoma cells to a normal morphogenic phenotype in a 3D culture. Proc Natl Acad Sci USA 2003; 100:521-526.
13 Nittka S, Gunther J, Ebisch C, Erbesdobler RA, Neumaier M: The human tumor suppressor CEACAM1 modulates apoptosis and is implicated in early colorectal tumorigenesis. Oncogene 2004; 23:9306-9313.

14 Nittka S, Bohm C, Zentgraf FH, Neumaier M: The CEACAM1-mediated apoptosis pathway is activated by CEA and triggers dual cleavage of CEACAM1. Oncogene 2008;27:3721-3728.

15 Neumaier M, Paululat S, Chan A, Matthaes P, Wagener C: Biliary glycoprotein, a potential human cell adhesion molecule, is down-regulated in colorectal carcinomas. Proc Natl Acad Sci USA 1993;90:10744-10748.

16 Nollau P, Prall F, Helmchen U, Wagener C, Neumaier M: Dysregulation of carcinoembryonic antigen group members CGM2, DC66a (biliary glycoprotein), and nonspecific cross-reacting antigen in colorectal carcinomas. Comparative analysis by Northern blot and in situ hybridization. Am J Pathol 1997;151:521-530.
17 Scholzel S, Zimmermann W, Schwarzkopf G, Grunert F, Rogaczewski B, Thompson J: Carcinoembryonic antigen family members CEACAM6 and CEACAM7 are differentially expressed in normal tissues and oppositely deregulated in hyperplastic colorectal polyps and early adenomas. Am J Pathol 200;156:595-605.

18 Chaves P, Crux C, Lage P, et al.: Immunhistochemical detection of mismatch repair gene proteins as a useful tool for the identification of colorectal carcinoma with the mutator phenotype. J Pathol 2000; 191:355-360.

19 Schiemann U, Gunther S, Gross M, et al.: Preoperative serum levels of the carcinoembryonic antigen in hereditary non-polyposis colorectal cancer compared to levels in sporadic colorectal cancer. Cancer Detect Prev 2005;29:356-360.

20 Sanchez-de-Abajo A, de la Hoya M, van Puijenbroek M, et al.: Molecular analysis of colorectal cancer tumors from patients with mismatch repair proficient hereditary nonpolyposis colorectal cancer suggests novel carcinogenic pathways. Clin Cancer Res 2007;13:5729-5735. 